\title{
Intralipid treatment for Alzheimer Disease
}

\author{
Joseph Eldor* \\ Theoretical Medicine Institute, Jerusalem, Israel
}

\begin{abstract}
Currently, there is no cure for Alzheimer's. But drug and non-drug treatments may help with both cognitive and behavioral symptoms. Researchers are looking for new treatments to alter the course of the disease and improve the quality of life for people with dementia.

Intralipid treatment is first suggested here for the treatment of Alzheimer disease. It should be given intravenously on a monthly basis according to each patient's response. Clinical studies should be done in order to evaluate this new treatment modality.
\end{abstract}

\section{Dr. Aloysius “Alois” Alzheimer}

Dr. Aloysius "Alois" Alzheimer was a German psychiatrist and neuropathologist and a colleague of Emil Kraepelin. Alzheimer is credited with identifying the first published case of "presenile dementia", which Kraepelin would later identify as Alzheimer's disease [1,2].

In 1901, Dr. Alzheimer observed a patient at the Frankfurt Asylum named Auguste Deter. The 51-year-old patient had strange behavioral symptoms, including a loss of short-term memory; she became his obsession over the coming years. Auguste Deter was a victim of the politics of the time in the psychiatric community; the Frankfurt asylum was too expensive for her husband. Mr. Deter made several requests to have his wife moved to a less expensive facility, but Dr. Alzheimer intervened in these requests. Ms. Deter remained at the Frankfurt asylum, where Alzheimer had made a deal to receive her records and brain upon her death [3]. On 8 April 1906, Ms. Deter died, and Dr. Alzheimer had her medical records and brain brought to Munich where he was working in Kraepelin's laboratory. With two Italian physicians, he used the staining techniques of Bielschowsky to identify amyloid plaques and neurofibrillary tangles. These brain anomalies would become identifiers of what later became known as Alzheimer's Disease [4].

\section{Alzheimer's disease}

Some evidence indicates that disruption of the blood-brain barrier in Alzheimer's disease patients allows blood plasma containing amyloid beta $(A \beta)$ to enter the brain where the $A \beta$ adheres preferentially to the surface of astrocytes [5]. These findings have led to the hypotheses that [1] breakdown of the blood-brain barrier allows access of neuronbinding autoantibodies and soluble exogenous $A \beta 42$ to brain neurons and [2] binding of these autoantibodies to neurons triggers and/or facilitates the internalization and accumulation of cell surface-bound A $\beta 42$ in vulnerable neurons through their natural tendency to clear surface-bound autoantibodies via endocytosis. Eventually the astrocyte is overwhelmed, dies, ruptures, and disintegrates, leaving behind the insoluble $\mathrm{A} \beta 42$ plaque. Thus, in some patients, Alzheimer's disease may be caused (or more likely, aggravated) by a breakdown in the bloodbrain barrier [6]. AD evolves with widespread loss of neurons and their synapses in such key brain areas as the cerebral cortex, entorhinal area, and hippocampus. At the gross level, this is evident as a general shrinkage of the brain away from the cranial vault and a corresponding dilation of the fluid-filled brain ventricles to fill the void. At the microscopic level, there are several different pathological changes that occur, but one consistent pathological hallmark is the early appearance of amyloid plaques. These plaques are abundant and widely scattered throughout $\mathrm{AD}$-vulnerable brain regions. They contain a 42 -amino acid protein fragment, known as amyloid 1-42 (A42), that is derived from the sequential enzymatic cleavage of the much larger amyloid precursor protein. Once produced, A42 has the ability to self-assemble into nondegradeable fibrils that can persist in $\mathrm{AD}$ brains long after the neurons in which they accumulated have died [7].

Alzheimer's disease (AD) was first described in 1906 by German psychiatrist Alois Alzheimer, who observed abnormal clumps and tangled bundles of protein in the brain of a patient who experienced memory loss, language difficulties, and abnormal behaviour [8]. The risk of developing $\mathrm{AD}$ increases exponentially with age and is the leading cause of dementia and the most common neurodegenerative disease in the elderly; prevalence rates in 65-74 years old are estimated to be $3 \%$, rising to $19 \%$ for $75-85$ years old, and nearly $50 \%$ in those aged over 85 (9). AD is more common among older people but it is not a normal part of ageing. As the global population ages, the prevalence of $\mathrm{AD}$ is expected to rise from 36 million to 115 million sufferers by 2050 [9].

It is estimated that over $5 \%$ of the U.S. population over 65 and over $15 \%$ of the U.S. population over 85 are beset with some form of Alzheimer's disease. It is believed that the principal cause for confinement of the elderly in long term care facilities is due to this disease, and approximately $65 \%$ of those dying in skilled nursing facilities suffer from it [10] intravenous lipid emulsion The idea that intravenous lipid emulsion could be used to affect the pharmacokinetics of a drug in circulation was first introduced fifty years ago. It was shown that rats infused lipid emulsion after an injection of the barbiturate thiopental emerged more rapidly from anaesthesia than rats infused the same

Correspondence to: Joseph Eldor, Theoretical Medicine Institute, Jerusalem, Israel, E-mail: csen_international@csen.com

Key words: Alzheimer disease, Intralipid

Received: June 20, 2017; Accepted: July 15, 2017; Published: July 17, 2017 
volume of fat-free solution [11]. Other early studies were published on the effect of lipid emulsion on chlorpromazine availability in rabbits [12], and the effect of lipid emulsion on the elimination of phenytoin [13]. Although the studies show some effect of lipid emulsion, this did not kindle more widespread interest in the subject. The serendipitous discovery of the apparently shielding effect of a large intravenous dose of lipid emulsion against bupivacaine toxicity in rats triggered renewed interest in the field [14]. Additional experimental animal and isolated heart studies were performed [15-16], and although efficacy and safety had not been established by clinical trials, clinicians soon applied lipid therapy to seemingly hopeless cases of severe intoxication [17].

Intravenous lipid emulsion therapy for severe intoxication is a relatively young field. Although a few early studies on the pharmacokinetic effects of intravenous lipid emulsion exist [11-13;18], its use as a treatment for severe intoxication was proposed as late as 1998 [14]. Since this proposal, no randomized controlled human trials have been published. Thus, the evidence supporting this use of lipid emulsion consists only of animal studies and human case reports of varying quality [20]. Amyloid beta-peptide (Abeta) is a key molecule in Alzheimer disease (AD). Cerebral deposition of Abeta was earlier thought to initiate the pathological cascade of $\mathrm{AD}$, including the formation of senile plaques and neurofibrillary tangles, neuronal loss, and dementia. According to the classical amyloid hypothesis, the aggregation of Abeta into insoluble beta- sheet fibrils plays an important role in its neurotoxicity. However, this hypothesis is paradoxical: The concentrations of Abeta required for fibrillization and neurotoxicity are higher than its physiological concentrations. Cognitive decline in $\mathrm{AD}$ patients is not correlated with the levels of senile plaque formation or insoluble Abeta formation; instead it correlates with the levels of synapse loss and the levels of soluble Abeta. These observations suggest the existence of soluble toxic forms of Abeta in $\mathrm{AD}$ brains; these forms have recently been identified to be oligomeric assemblies of Abeta. At present, $\mathrm{AD}$ is believed to begin with synaptic dysfunction caused by soluble Abeta oligomers. This hypothesis termed the oligomer hypothesis, is based on the following observations: The levels of Abeta oligomers are high in $\mathrm{AD}$ brains. Exogenous Abeta oligomers at physiological concentrations cause synaptic and cognitive dysfunction in vivo and synapse loss and neuronal death in vitro. Furthermore, we observed that the E693delta mutation in the amyloid precursor protein found in $\mathrm{AD}$ patients causes disease by increasing the formation of Abeta oligomers without inducing the formation of Abeta fibrils or senile plaques. Currently, senile plaque formation is thought to occur in order to protect neurons from the toxicity of diffusible Abeta oligomers by sequestering them into deposits. Thus, soluble Abeta oligomers play a more important role in the etiology of $\mathrm{AD}$ insoluble Abeta fibrils [21].

The defining features of Alzheimer disease (AD) include conspicuous changes in both brain histology and behavior. The $\mathrm{AD}$ brain is characterized microscopically by the combined presence of 2 classes of abnormal structures, extracellular amyloid plaques and intraneuronal neurofibrillary tangles, both of which comprise highly insoluble, densely packed filaments. The soluble building blocks of these structures are amyloid- $\beta(\mathrm{A} \beta)$ peptides for plaques and tau for tangles. Amyloid- $\beta$ peptides are proteolytic fragments of

the transmembrane amyloid precursor protein, whereas tau is a brain-specific, axon-enriched microtubule-associated protein. The behavioral symptoms of $\mathrm{AD}$ correlate with the accumulation of plaques and tangles, and they are a direct consequence of the damage and destruction of synapses that mediate memory and cognition. Synapse loss can be caused by the failure of live neurons to maintain functional axons and dendrites or by neuron death.
During the past dozen years, a steadily accumulating body of evidence has indicated that soluble forms of $A \beta$ and tau work together, independently of their accumulation into plaques and tangles, to drive healthy neurons into the diseased state and that hallmark toxic properties of $A \beta$ require tau. For instance, acute neuron death, delayed neuron death following ectopic cell cycle reentry, and synaptic dysfunction are triggered by soluble, extracellular $A \beta$ species and depend on soluble, cytoplasmic tau. Therefore, $A \beta$ is upstream of tau in $\mathrm{AD}$ pathogenesis and triggers the conversion of tau from a normal to a toxic state, but there is also evidence that toxic tau enhances $A \beta$ toxicity via a feedback loop. Because soluble toxic aggregates of both $A \beta$ and tau can self-propagate and spread throughout the brain by prionlike mechanisms, successful therapeutic intervention for $\mathrm{AD}$ would benefit from detecting these species before plaques, tangles, and cognitive impairment become evident and from interfering with the destructive biochemical pathways that they initiate [22].

The increasing prevalence of Alzheimer's disease (AD) and a lack of effective prevention or disease-modifying therapies are global challenges with devastating personal, social and economic consequences. The amyloid $\beta(\mathrm{A} \beta)$ hypothesis posits that cerebral $\beta$-amyloidosis is a critical early event in AD pathogenesis. However, failed clinical trials of $\mathrm{A} \beta$-centric drug candidates have called this hypothesis into question. Whereas we acknowledge that the $A \beta$ hypothesis is far from disproven, we here re-visit the links between $A \beta$, tau and neurodegeneration. We review the genetics, epidemiology and pathology of sporadic $\mathrm{AD}$ and give an updated account of what is currently known about the molecular pathogenesis of the disease [23]. The amyloid hypothesis has driven drug development strategies for Alzheimer's disease for over 20 years. We review why accumulation of amyloid-beta $(A \beta)$ oligomers is generally considered causal for synaptic loss and neurodegeneration in AD. We elaborate on and update arguments for and against the amyloid hypothesis with new data and interpretations, and consider why the amyloid hypothesis may be failing therapeutically. We note several unresolved issues in the field including the presence of $A \beta$ deposition in cognitively normal individuals, the weak correlation between plaque load and cognition, questions regarding the biochemical nature, presence and role of $A \beta$ oligomeric assemblies in vivo, the bias of pre-clinical AD models toward the amyloid hypothesis and the poorly explained pathological heterogeneity and comorbidities associated with $\mathrm{AD}$. We also illustrate how extensive data cited in support of the amyloid hypothesis, including genetic links to disease, can be interpreted independently of a role for $\mathrm{A} \beta$ in $\mathrm{AD}$. We conclude it is essential to expand our view of pathogenesis beyond $A \beta$ and tau pathology and suggest several future directions for $\mathrm{AD}$ research, which we argue will be critical to understanding $\mathrm{AD}$ pathogenesis [24].

The aggregation and deposition of amyloid-beta (Abeta) in the brain is thought to be an early event in the pathology of Alzheimer's disease (AD). Many studies have reported the association of Abeta with lipoproteins from plasma suggesting an involvement of lipoprotein particles in Abeta transport. Chylomicron-like lipid emulsions, resembling chylomicrons in composition, size and metabolism were prepared in the presence of [125I] Abeta1-40. Abeta was found to associate significantly with these lipid emulsions during their preparation. The chylomicron-like emulsions containing Abeta were then injected into a lateral ear vein of conscious rabbits and blood sampled at regular intervals up to 30 mins. It was observed that there was no difference in the plasma clearance of [125I] Abeta and that of the $3 \mathrm{H}$-cholesteryl ester, a marker of the emulsion particles, demonstrating that Abeta remains associated with these particles throughout both their lipolysis and tissue uptake. Our results show that Abeta can be 
metabolised in association with triglyceride rich lipoproteins (TRLs). In addition we report the presence of specific markers of TRLs of hepatic and intestinal origin in human CSF thus suggesting a potential means of cerebral Abeta delivery[25].

Docosahexaenoic acid (DHA), the main $n-3$ polyunsaturated fatty acid (PUFA) in membranes, is particularly abundant in brain cells. Decreased cerebral concentrations of DHA, resulting from dietary n-3 deficiency, are associated with impaired cognitive function. Because the cellular causes of this impairment are still unknown, we need in vitro models that mimic the variations in $n-3 / n-6$ PUFA seen in vivo. We have compared the PUFA profiles of hamster astrocytes cultured in medium supplemented with long- chain PUFA [DHA and/or arachidonic acid (AA)] with those of brain tissue from hamsters fed an n-6/n-3 PUFA-balanced diet or one lacking n-3 PUFA. Astrocytes were obtained from the brain cortex of newborn hamsters and cultured in minimum essential medium $+5 \%$ fetal calf serum (FCS) supplemented with DHA and/or AA for 10 days. The astrocytes cultured in medium + FCS had low n-3 PUFA contents, comparable to those of brain tissue from hamsters fed an n-3-deficient diet. We have shown that astrocytes grown in medium supplemented with DHA and/or AA, plus alphatocopherol to prevent lipid peroxidation, incorporated large amounts of these long-chain PUFA, so that the n-6/n-3 PUFA compositions of the phosphatidylethanolamine and phosphatidylcholine, the two main classes of membrane phospholipids, were greatly altered. Astrocytes cultured in medium plus DHA had a more physiological n-3 status, grew better, and retained their astrocyte phenotype. Thus astrocytes in culture are likely to be physiologically relevant only when provided with adequate DHA. This reliable method of altering membrane phospholipid composition promises to be useful for studying the influence of n-6/n-3 imbalance on astrocyte function [26].

Rat neural stem cells/neural progenitors (NSC/NP) are generally grown in serum-free medium. In this study, NSC/NP were supplemented with the main long-chain polyunsaturated fatty acids (PUFAs) present in the brain, arachidonic acid (AA), or docosahexaenoic acid (DHA), and were monitored for their growth. Lipid and fatty acid contents of the cells were also determined. Under standard conditions, the cells were characterized by phospholipids displaying a highly saturated profile, and very low levels of PUFAs. When cultured in the presence of PUFAs, the cells easily incorporated them into the phospholipid fraction. We also compared the presence of three membrane proteins in the lipid raft fractions: GFR and connexin 43 contents in the rafts were increased by DHA supplementation, whereas Gbeta subunit content was not significantly modified. The restoration of DHA levels in the phospholipids could profoundly affect protein localization and, consequently, their functionalities [27].

Lipids are the fundamental structural components of biological membranes. For a long time considered as simple barriers segregating aqueous compartments, membranes are now viewed as dynamic interfaces providing a molecular environment favorable to the activity of membrane-associated proteins. Interestingly, variations in membrane lipid composition, whether quantitative or qualitative, play a crucial role in regulation of membrane protein functionalities. Indeed, a variety of alterations in brain lipid composition have been associated with the processes of normal and pathological aging.

Although not establishing a direct cause-and-effect relationship between these complex modifications in cerebral membranes and the process of cognitive decline, evidence shows that alterations in membrane lipid composition affect important physicochemical properties notably impacting the lateral organization of membranes, and thus microdomains. It has been suggested that preservation of microdomain functionality may represent an effective strategy for preventing or decelerating neuronal dysfunction and cerebral vulnerability, processes that are both aggravated by aging. The working hypothesis developed in this review proposes that preservation of membrane organization, for example, through nutritional supplementation of docosahexaenoic acid, could prevent disturbances in and preserve effective cerebral function [28]. To date, no study has been performed to evaluate the antidotal effect of intravenous lipid emulsion on the poisoned patients' level of consciousness and routine metabolic profile tests in non-local anesthetic drug overdose. Taftachi F. et al. [29] aim was to evaluate the effect of intravenous intralipid administration as an antidote on the poisoned patients' Glasgow Coma Scale (GCS), hemodynamic parameters, arterial blood gas analysis, and routine metabolic profile tests (i.e., urea, glucose, sodium, and potassium) in the setting of non-local anesthetic drug overdose.

In this randomized controlled trial, a total of 30 patients with nonlocal anesthetic drug intoxication were enrolled and randomly assigned into case $(n=15)$ and control $(n=15)$ groups. In the case group, all patients received $10 \mathrm{cc} / \mathrm{kg}$ intralipid $10 \%$ infusion. The patients in the control group just received the supportive care. Patients' demographic and clinical characteristics and results of their laboratory tests were evaluated at presentation and 6 hours after that. Mean age was $23+/-5$ and $28+/-11$ years in cases and controls, respectively. There were no significant statistical differences between these two groups regarding age, gender, elapsed time between intubation and extubation, and need for intubation and/or mechanical ventilation $(\mathrm{p}=0.70$ and $\mathrm{p}=$ 1.00 , respectively). Also, systolic blood pressure, pulse rate, mean rate pressure product, respiratory rate, results of arterial blood gas analyses, serum sodium, potassium, urea, and creatinine on presentation and six hours later were not statistically significantly different between the two study groups. However, a significant difference was found between the two groups in terms of GCS difference $(p=0.048)$ and blood glucose six hours after presentation $(\mathrm{p}=0.04)$.

In the setting of non-local anesthetic drug overdose, intravenous intralipid infusion can increase GCS and interestingly, decrease the blood glucose. Lipid emulsions are widely used as carriers for hypnotics such as propofol, etomidate, and diazepam. It is assumed that the emulsions alone exert no effect on cellular functions nor influence the pharmacokinetics, pharmacodynamics, or anesthetic and analgetic potency of the hypnotics they carry. To elucidate possible interactions between lipid emulsions and cell membranes, in particular membrane-bound proteins, we investigated the effects of commercially available lipid emulsions on the cell membranes of cultured cortical neurons from the mouse by using the whole-cell configuration of the patch-clamp technique. Of nine lipid emulsions tested, three, i.e., Intralipid, Structolipid, and, to a much lesser extent, Abbolipid, activated membrane currents in the neuronal cells in a dilution-dependent manner. The emulsion-induced currents were not affected by picrotoxin or bicuculline but were inhibited by DL-AP 5 and ketamine. The voltage dependence of the currents was influenced by the presence of $\mathrm{Mg}\left(2^{+}\right)$in a way that is typical for currents conducted by $\mathrm{N}$-methyl-D-aspartate receptor channels. We conclude that Intralipid, Structolipid, and Abbolipid activate N-methyl-D-aspartate receptor channels in cortical neurons.

Lipid emulsions are widely used as carriers for hypnotics such as propofol, etomidate, or diazepam. We tested nine commercially available lipid emulsions and demonstrate that three of them-Intralipid, Structolipid, and Abbolipid--activate NMDA receptor 
channels in the membranes of cortical neuronal cells [30] Haywood SC et al. [31] tested the hypothesis that lipids could act as an alternative fuel source in the brain during insulin-induced hypoglycemia. Male Sprague-Dawley rats were subjected to hyperinsulinemic (5 $\mathrm{mU} \cdot \mathrm{kg}^{-1} \cdot \mathrm{min}^{-1}$ hypoglycemic (approximately $50 \mathrm{mg} / \mathrm{dl}$ ) clamps. In protocol1, intralipid (IL), a fat emulsion, was infused intravenously to prevent the fall in free fatty acid levels that occurs in response to hyperinsulinemic hypoglycemia. Intravenous lipid infusion did not alter the counterregulatory responses to hypoglycemia. To test whether IL could have central effects in mediating the counterregulatory response to hypoglycemia, in protocol 2 the brains of precannulated rats were intracerebroventricularly (icv) infused with IL or artificial cerebrospinal fluid (aCSF) as control. Unexpectedly, the epinephrine and glucagon response to hypoglycemia was significantly augmented with icv IL infusion. To determine whether central IL infusion could restore defective counterregulation, in protocol 3 rats were made recurrently hypoglycemic (RH) for 3 days and on the 4th day underwent hyperinsulinemic hypoglycemic clamps with icv IL or aCSF infusion. $\mathrm{RH}$ rats had the expected impaired epinephrine response to hypoglycemia, and icv IL infusion again significantly augmented the epinephrine response in $\mathrm{RH}$ rats to normal. With regard to our experimental model of hypoglycemic counterregulation, we conclude that 1) systemic lipid infusion did not alter the counterregulatory response to hypoglycemia, 2) the icv infusion of lipids markedly increased CSF FFA levels and paradoxically augmented the epinephrine and glucagon responses, and 3) the blunted sympathoadrenal response in recurrently hypoglycemic rats was completely normalized with the icv lipid infusion. It is concluded that, in the setting of insulin-induced hypoglycemia, increased brain lipids can enhance the sympathoadrenal response. Lipid infusion reverses systemic local anesthetic toxicity. The acceptable upper limit for lipid administration is unknown and has direct bearing on clinical management. We hypothesize that high volumes of lipid could have undesirable effects and sought to identify the dose required to kill $50 \%$ of the animals (LD (50)) of large volume lipid administration. Intravenous lines and electrocardiogram electrodes were placed in anesthetized, male Sprague-Dawley rats. Twenty percent lipid emulsion $(20,40,60$, or $80 \mathrm{~mL} / \mathrm{kg}$ ) or saline (60 or $80 \mathrm{~mL} / \mathrm{kg}$ ), were administered over 30 mins; lipid dosing was assigned by the Dixon "up-and-down" method. Rats were recovered and observed for $48 \mathrm{hrs}$ then euthanized for histologic analysis of major organs. Three additional rats were administered $60 \mathrm{~mL} / \mathrm{kg}$ lipid emulsion and euthanized at 1,4 , and 24 hrs to identify progression of organ damage.

The maximum likelihood estimate for LD (50) was 67.72 (SE, 10.69) $\mathrm{mL} / \mathrm{kg}$. Triglycerides were elevated immediately after infusion but returned to baseline by $48 \mathrm{hrs}$ when laboratory abnormalities included elevated amylase, aspartate aminotransferase, and serum urea nitrogen for all lipid doses. Histologic diagnosis of myocardium, brain, pancreas, and kidneys was normal at all doses. Microscopic abnormalities in lung and liver were observed at 60 and $80 \mathrm{~mL} / \mathrm{kg}$; histopathology in the lung and liver was worse at $1 \mathrm{hr}$ than at 4 and $24 \mathrm{hrs}$.

The LD (50) of rapid, high volume lipid infusion is an order of magnitude greater than doses typically used for lipid rescue in humans and supports the safety of lipid infusion at currently recommended doses for toxin-induced cardiac arrest. Lung and liver histopathology was observed at the highest infused volumes [32]. Intravenous lipid emulsion has been suggested as treatment for severe intoxications caused by lipophilic drugs, including tricyclic antidepressants. We investigated the effect of lipid infusion on plasma and tissue concentrations of amitriptyline and haemodynamic recovery, when lipid was given after amitriptyline distribution into well-perfused organs. Twenty anaesthetized pigs received amitriptyline intravenously $10 \mathrm{mg} / \mathrm{kg}$ for $15 \mathrm{~min}$. Thirty minutes later, in random fashion, $20 \%$ Intralipid $\left({ }^{\circ}\right)$ (Lipid group) or Ringer's acetate (Control group) was infused $1.5 \mathrm{ml} / \mathrm{kg}$ for $1 \mathrm{~min}$. followed by $0.25 \mathrm{ml} / \mathrm{kg} / \mathrm{min}$. for 29 min. Arterial and venous plasma amitriptyline concentrations and haemodynamics were followed till $75 \mathrm{~min}$. after amitriptyline infusion. Then, frontal brain and heart apex samples were taken for amitriptyline measurements. Arterial plasma total amitriptyline concentrations were higher in the Lipid than in the Control group $(\mathrm{p}<0.03)$ from 20 min. on after the start of the treatment infusions. Lipid emulsion reduced brain amitriptyline concentration by $25 \%(\mathrm{p}=0.038)$ and amitriptyline concentration ratios brain/arterial plasma $(\mathrm{p}=0.016)$ and heart/arterial plasma $(\mathrm{p}=0.011)$. There were no differences in ECG parameters and no severe cardiac arrhythmias occurred. Two pigs developed severe hypotension during the lipid infusion and were given adrenaline. In conclusion, lipid infusion, given not earlier than after an initial amitriptyline tissue distribution, was able to entrap amitriptyline back into plasma from brain and possibly from other highly perfused, lipid-rich tissues. In spite of the entrapment, there was no difference in haemodynamics between the groups [33]. Malathion is one of the most widely used organophosphate pesticides and herbicides. It has given rise to major clinical problems by its poisoning in all over the world. Malathion also a highly lipophilic agent, and tends to accumulate within lipid-rich tissue like a brain in the body, causing toxicity. Therefore, the study was aimed to investigate if there is a possible beneficial effect of using intralipid fat emulsion (IFE) on the neurotoxicity, and to detect it time-dependently at the beginning, 6th and 12th hours of M intoxication. Forty-eight rats were randomly divided into six groups including: control (C), Lipid (L) group (18.6 $\mathrm{mL} / \mathrm{kg}$ oral IFE), Malathion (M) group (10 mg/kg oral M), M0L group (IFE treated after immediate from M), M6L group (IFE treated after 6 hours from M), M12L group (IFE treated after 12 hours from M). M group in comparison with all others group, there was an increase in the total oxidant status (TOS) level. M group in comparison with C, L, MOL groups, it was seen significantly decrease in the total antioxidant capacity (TAC) level. Interestingly, M group in comparison with M6L and M12L groups, there was no significant difference among these groups in terms of the TAC levels. Although there was no significant difference among $\mathrm{C}, \mathrm{L}$ and MOL groups in terms of both TAC and TOS levels, but was significant difference $\mathrm{C}$, $\mathrm{L}$ groups in comparison with M6L, M12L groups in terms of TAC levels. C group in comparison with L, M0L, M6L, M12L groups in terms of TOS levels, there was no significant difference. These findings have indicated that IFE seriously reduced TOS levels in all the groups depending on time. Also, M0L group in comparison with M6L and M12L groups, there was significantly increase of the TAC levels. There was no statistically significant difference between M6L and M12L groups. These biochemical results were confirmed with immunohistochemical results.

The study has had some certain evidence that IFE is a promising safe therapy for acutely intoxicated cases by organophosphate. It is much more effective if used at the beginning of organophosphate poisoning. As such, there is no need to avoid using IFE in clinical practice [34].

Chlorpyriphos is one of the most widely used organophosphate (OP) insecticide in agriculture with potential toxicity. Current postexposure treatments consist of anti-cholinergic drugs and oxime compounds. We studied the effects of intralipid and caffeic acid phenethyl ester (CAPE) on chlorpyriphos toxicity to compose an alternative or supportive treatment for OP poisoning. 
Forty-nine rats were randomly divided into seven groups. Chlorpyriphos was administered for toxicity. Intralipid (IL) and CAPE administered immediately after chlorpyriphos. Serum acetylcholinesterase (AChE) level, total oxidant status (TOS), total antioxidant response (TAR), and histologic examination of cerebellum and brain tissue with Hematoxylin-Eosin and immunohistochemical dyes were examined.

Serum enzym levels showed that chlorpyriphos and CAPE inhibited AChE while IL alone had no effect, chlorpyriphos and CAPE intensifies the inhibition effect. Significant difference at AChE levels between the chlorpyriphos+IL and chlorpyriphos+CAPE verified that IL has a protective effect on AChE inhibition. TAR levels were significantly increased in all groups except chlorpyriphos group, TOS levels revealed that CAPE and IL decrease the amount of oxidative stress. Histologic examination revealed that neuronal degeneration was slightly decreased at chlorpyriphos+IL group, but CAPE had a significant effect on protection of neuronal degeneration.

The results of this study gave us three key points. 1) AChE activity is important for diagnosis of OP intoxication but it has no value for determining the neuro-degeneration. 2) CAPE inhibits AChE activity and may increase the muscarinic-nicotinic hyperactivation. Therefore it should not be used for treatment of OP intoxication. 3) IL decreases the severity of neurodegeneration and symptoms of OP intoxication and it can be used as a supportive agent [35].

Intravenous lipid emulsion has been suggested as treatment for local anaesthetic toxicity, but the exact mechanism of action is still uncertain. Controlled studies on the effect of lipid emulsion on toxic doses of local anaesthetics have not been performed in man. In randomized, subjectblinded and two-phase cross-over fashion, eight healthy volunteers were given a $1.5 \mathrm{ml} / \mathrm{kg}$ bolus of $20 \%$ Intralipid( $\left.{ }^{\circ}\right)(200 \mathrm{mg} / \mathrm{ml})$ or Ringer's acetate solution intravenously, followed by a rapid injection of lidocaine $1.0 \mathrm{mg} / \mathrm{kg}$. Then, the same solution as in the bolus was infused at a rate of $0.25 \mathrm{ml} / \mathrm{kg} / \mathrm{min}$. for $30 \mathrm{~min}$. Electroencephalography (EEG) was recorded, and $5 \mathrm{~min}$. after lidocaine injection, the volunteers were asked to report subjective symptoms. Total and un-entrapped lidocaine plasma concentrations were measured from venous blood samples. EEG band power changes (delta, alpha and beta) after the lidocaine bolus were similar during lipid and during Ringer infusion. There were no differences between infusions in the subjective symptoms of central nervous system toxicity. Lidocaine was only minimally entrapped in the plasma by lipid emulsion, but the mean un-entrapped lidocaine area under concentration-time curve from 0 to $30 \mathrm{~min}$. was clearly smaller during lipid than Ringer infusion (16.4 versus $21.3 \mathrm{mg} \times \mathrm{min} / \mathrm{l}$, $\mathrm{p}=0.044)$. Intravenous lipid emulsion did not influence subjective toxicity symptoms nor affect the EEG changes caused by lidocaine [36].

\section{Intralipid treatment for Alzheimer Disease}

Lange DB et al. [37] described a case of severe central nervous system toxicity after an overdose of lidocaine by local infiltration in a peritoneal dialysis patient and subsequent treatment of the toxicity with lipid emulsion. A 31-year-old male received an iatrogenic overdose of $1600 \mathrm{mg}$ of lidocaine $2 \%$ by infiltration during an attempt to remove and replace a peritoneal dialysis catheter. Within 10 minutes after the last lidocaine injection, the patient exhibited features of local anesthetic toxicity, which included tachycardia, hypertension, shortness of breath, dizziness, and a choking sensation that progressed to hallucinations, dysarthria, and uncoordinated, weak limb movement. Within 10 minutes after administration of a single $1.5-\mathrm{mg} / \mathrm{kg}$ intravenous bolus of $1.5 \mathrm{~mL} / \mathrm{kg}$ [corrected], the patient improved dramatically. After observation overnight in a monitored care setting, the patient was discharged home with no apparent neurologic sequelae. Systemic toxicity due to regional anesthesia with local anesthetic agents such as lidocaine has been well described in the medical literature. The use of lipid emulsion as an antidote to the toxicity of local anesthetics and other lipophilic drugs has been suggested as a valuable intervention in both early, rapidly progressive toxicity, as well as toxicity that is refractory to standard treatment. Patients with advanced chronic kidney disease may be more susceptible to systemic effects of lidocaine due to decreased drug elimination.

Central nervous system toxicity due to an overdose of lidocaine was quickly reversed by intravenous lipid emulsion in our patient [37]. The major component fatty acids in Intralipid are linoleic acid (44-62\%), oleic acid (19-30\%), palmitic acid (7-14\%), a-linolenic acid (4-11\%) and stearic acid (1.4-5.5\%) [37]. Oxidative stress is a hallmark of many degenerative disorders. The brain is particularly vulnerable to this phenomenon owing to high oxygen consumption, enrichment in polyunsaturated fatty acids (PUFAs) and high levels in redox metal ions [38]. Lipid peroxidation products (LPPs) have been found in brain, cerebrospinal fluid and plasma from patients with Alzheimer's disease (AD)/t [38]. Primary substrates for lipid peroxidation are PUFAs and include $\omega-6$ fatty acids (for example, linoleic acid and arachidonic acid) as well as $\omega-3$ fatty acids (for example, docosahexaenoic acid). Reactive oxygen species are responsible for starting the chain by the production of an unstable lipid radical that is converted to a lipid peroxyl radical, leading to the peroxidation of other fatty acids (propagation). This chain reaction stops (termination) when two radicals react to produce a non-radical species, or as a result of antioxidants (for example, vitamin $\mathrm{C}$ and vitamin E) and enzymes of the superoxide dismutase, catalase and peroxidase families [38]. Oxidized PUFAs are further degraded to toxic products, such as 4-hydroxy-2- nonenal (HNE), acrolein and other short-chain aldehydes. Importantly, amyloid- $\beta$ has been shown to cause oxidative stress through its interaction with transition metal ions, such as $\mathrm{Cu} 2+$ and $\mathrm{Zn} 2+$, which are enriched in senile plaques [39]. Amyloid- $\beta$ can reduce these metal ions, thus producing hydrogen peroxide. During this process, amyloid- $\beta$ becomes oxidized, thereby leading to the crosslinking of some of its residues' side-chains and the formation of aggregate-prone adducts. Alternatively, hydrogen peroxide can be generated catalytically from $\mathrm{Cu} 2+-$ or $\mathrm{Zn} 2+$-bound amyloid- $\beta$ using other electron donors (for example, PUFAs and cholesterol), a process leading to the generation of toxic LPPs, such as oxysterol and HNE. Finally, amyloid- $\beta$ itself can be crosslinked by HNE. Key challenges in the field are to understand the role of LPP accumulation in the progression of $\mathrm{AD}$-associated manifestations.

The linoleic acid metabolism was examined in the brain cortex of 4 month-old and 24 month-old rats. After the injection of [1-14C]linoleate into the lateral ventricle of the brain the animals were sacrificed at 1,3 and 6 hours from the injection. The linoleate (18:2) incorporation into lipids, the presence of fatty acid peroxidation products, as well as the 18:2 transformation into elongated and desaturated derivatives were determined. Both an age-related reduction in linoleate incorporation rate into glycerophospholipids and a decrease in fatty acid turnover were found. Furthermore, in glycerophospholipids from 24 month-old rat brain cortex a higher level of hydroperoxide derivative of linoleate was found as compared to 4 month-old animals, and this damaged fatty acid is eliminated more slowly in aged rats than in adults. Finally, unlike 4 month-old animals, a stimulation of the transformation rate of linoleate into desaturation $(6,9,12-\mathrm{C} 18: 3)$ and elongation $(8,11,14$, C20:3) products was found in 24 month-old rat brain cortex. On the 
contrary, as far as arachidonic acid (one of the most important end products of the mechanism of linoleate modification) is concerned, the differences between aged and control animals were small, making it quite difficult to attribute a physiological meaning to this phenomenon [40].

Alzheimer's disease and associated diseases constitute a major public health concern worldwide. Nutrition-based, preventive strategies could possibly be effective in delaying the occurrence of these diseases and lower their prevalence. Arachidonic acid is the second major polyunsaturated fatty acid (PUFA) and several studies support its involvement in Alzheimer's disease. The objective of this review is to examine how dietary arachidonic acid contributes to Alzheimer's disease mechanisms and therefore to its prevention. First, we explore the sources of neuronal arachidonic acid that could potentially originate from either the conversion of linoleic acid, or from dietary sources and transfer across the blood-brain-barrier. In a second part, a brief overview of the role of the two main agents of Alzheimer's disease, tau protein and $\mathrm{A} \beta$ peptide is given, followed by the examination of the relationship between arachidonic acid and the disease. Third, the putative mechanisms by which arachidonic acid could influence Alzheimer's disease occurrence and evolution are presented. The conclusion is devoted to what remains to be determined before integrating arachidonic acid in the design of preventive strategies against Alzheimer's disease and other neurodegenerative diseases [41].

Insulin resistance and type 2 diabetes are associated with an increased risk of neurodegenerative diseases. Brain-derived neurotrophic factor (BDNF) regulates neuronal differentiation and synaptic plasticity, and its decreased levels are supposed to play a role in the pathogenesis of Alzheimer's disease and other disorders. The aim of the current study was to estimate the effects of hyperinsulinemia and serum free fatty acids (FFA) elevation on circulating BDNF concentration in humans.

We studied 18 healthy male subjects (mean age $25.6 \pm 3.0$ years; mean BMI $26.6 \pm 4.8 \mathrm{~kg} / \mathrm{m}^{2}$. Serum and plasma BDNF concentration was measured in the baseline state and in the 120 and $360 \mathrm{~min}$ of euglycemic hyperinsulinemic clamp with or without intralipid/heparin infusion. Furthermore, plasma BDNF was measured in 20 male subjects (mean age $22.7 \pm 2.3$ years; mean BMI $24.9 \pm 1.5 \mathrm{~kg} / \mathrm{m}^{2} 360$ min after a high- fat meal. Insulin sensitivity was reduced by $\sim 40 \%$ after 6 h of intralipid/heparin infusion $(\mathrm{P}<0.001)$. During both clamps, serum and plasma BDNF followed the same pattern. Hyperinsulinemia had no effect on circulating BDNF. Raising FFA had no effect on circulating BDNF in $120 \mathrm{~min}$; however, it resulted in a significant decrease by $43 \%$ in serum and by $35 \%$ in plasma BDNF after $360 \mathrm{~min}(\mathrm{P}=0.005$ and 0.006 , respectively). High-fat meal also resulted in a decrease by $27.8 \%$ in plasma $\mathrm{BDNF}(\mathrm{P}=0.04)$.

Our data show that raising FFA decreases circulating BDNF. This might indicate a potential link between FFA-induced insulin resistance and neurodegenerative disorders [42]. Rodriguez-Navas C et al. [43] analyzed the fatty acid profile of brains and plasma from male and female mice fed chow or a western-style high fat diet (WD) for 16 weeks to determine if males and females process fatty acids differently. Based on the differences in fatty acids observed in vivo, we performed in vitro experiments on N43 hypothalamic neuronal cells to begin to elucidate how the fatty acid milieu may impact brain inflammation.

Using a comprehensive mass spectrometry fatty acid analysis, which includes a profile for 52 different fatty acid isomers, we assayed the plasma and brain fatty acid composition of age-matched male and female mice maintained on chow or a WD. Additionally, using the same techniques, we determined the fatty acid composition of N43 hypothalamic cells following exposure to palmitic and linoleic acid, alone or in combination.

The data demonstrate there is a sexual dimorphism in brain fatty acid content both following the consumption of the chow diet, as well as the WD, with males having an increased percentage of saturated fatty acids and reductions in $\omega 6$-polyunsaturated fatty acids when compared to females. Interestingly, we did not observe a sexual dimorphism in fatty acid content in the plasma of the same mice. Furthermore, exposure of $\mathrm{N} 43$ cells to the $\omega 6$-PUFA linoleic acid, which is higher in female brains when compared to males, reduces palmitic acid-induced inflammation.

The data suggest male and female brains, and not plasma, differ in their fatty acid profile. This is the first time, to our knowledge, lipidomic analyses has been used to directly test the hypothesis there is a sexual dimorphism in brain and plasma fatty acid composition following consumption of the chow diet, as well as following exposure to the WD.

\section{Blood-brain barrier}

Treatment strategies for Alzheimer's disease (AD) are still elusive. Thus, new strategies are needed to understand the pathogenesis of $\mathrm{AD}$ in order to provide suitable therapeutic measures. Available evidences suggest that in $\mathrm{AD}$, passage across the blood-brain barrier (BBB) and transport exchanges for amyloid- $\beta$-peptide (ABP) between blood and the central nervous system (CNS) compartments play an important regulatory role for the deposition of brain $\mathrm{ABP}$. New evidences suggest that $\mathrm{BBB}$ is altered in $\mathrm{AD}$. Studies favoring transport theory clearly show that $\mathrm{ABP}$ putative receptors at the $\mathrm{BBB}$ control the level of soluble isoform of $A B P$ in brain. This is achieved by regulating influx of circulating ABP into brain via specific receptor for advanced glycation end products (RAGE) and gp330/megalin-mediated transcytosis. On the other hand, the efflux of brain-derived ABP into the circulation across the vascular system via $\mathrm{BBB}$ is accomplished by low-density receptor-related protein-1 (LRP1). Furthermore, an increased BBB permeability in $\mathrm{AD}$ is also likely since structural damage of endothelial cells is quite frequent in $\mathrm{AD}$ brain. Thus, enhanced drug delivery in $\mathrm{AD}$ is needed to induce neuroprotection and therapeutic success. For this purpose, nanodrug delivery could be one of the available options that require active consideration for novel therapeutic strategies to treat $\mathrm{AD}$ cases. This review is focused on these aspects and provides new data showing that $\mathrm{BBB}$ plays an important role in $\mathrm{AD}$-induced neurodegeneration and neurorepair [44].

The blood-brain barrier (BBB) is a tightly regulated barrier in the central nervous system. Though the BBB is thought to be intact during neurodegenerative diseases such as Alzheimer's (AD) and Parkinson's disease (PD), recent evidence argues otherwise.

Dysfunction of the BBB may be involved in disease progression, eliciting of peripheral immune response, and, most importantly, altered drug efficacy. In this review, we will give a brief overview of the $\mathrm{BBB}$, its components, and their functions. We will critically evaluate the current literature in $\mathrm{AD}$ and $\mathrm{PD} \mathrm{BBB}$ pathology resulting from insult, neuroinflammation, and neurodegeneration. Specifically, we will discuss alterations in tight junction, transport and endothelial cell surface proteins, and vascular density changes, all of which result in altered permeability. Finally, we will discuss the implications of BBB dysfunction in current and future therapeutics. Developing a better appreciation of $\mathrm{BBB}$ dysfunction in $\mathrm{AD}$ and $\mathrm{PD}$ may not only provide novel strategies in treatment, but will prove an interesting milestone in understanding neurodegenerative disease etiology and progression [45]. 
It is not clear whether Alzheimer's Disease (AD) is primarily a neurodegenerative disorder or not. A body of evidence suggests that vascular disorder in brains of individuals with $\mathrm{AD}$ contributes to the extremes of this disease. This raises a question whether Alzheimer's dementia is secondary to vascular dysfunction in the central nervous system (CNS) and, therefore, the neurodegeneration that follows is a consequence of inadequate cerebral blood flow, altered brain metabolism and failure in physiological functions of brain endothelium which represents a site at the blood-brain barrier (BBB). In this paper the evidence for a primary role of the CNS vascular system in pathogenesis of Alzheimer's dementia is reviewed to show how alterations in transport across the $\mathrm{BBB}$ contribute to development of cerebral beta-amyloidosis in $\mathrm{AD}$. In addition, vascularly-based therapeutic strategies to limit the development of beta-amyloidosis and to remove amyloid and plaques from the CNS of $\mathrm{AD}$ individuals are discussed [46]. Protection of the brain is strengthened by active transport and $\mathrm{ABC}$ transporters. P-glycoprotein (P-gp) at the bloodbrain barrier (BBB) functions as an active efflux pump by extruding a substrate from the brain, which is important for maintaining loco-regional homeostasis in the brain and protection against toxic compounds. Importantly, dysfunctional BBB P-gp transport is postulated as an important factor contributing to accumulation of aggregated protein in neurodegenerative disorders such as Alzheimer's disease (AD) and Parkinson's disease (PD). Furthermore, P-gp is a major factor in mediating resistance to brain entry of numerous exogenous compounds, including toxins that can be involved in PD pathogenesis. This review highlights the role of altered P-gp function in the pathogenesis and progression of neurodegenerative disease. Also the implications of alterations in P-gp function for the treatment of these diseases are discussed [47].

The blood-brain barrier $(\mathrm{BBB})$ is a tightly regulated barrier in the central nervous system. Though the BBB is thought to be intact during neurodegenerative diseases such as Alzheimer's (AD) and Parkinson's disease (PD), recent evidence argues otherwise.

Dysfunction of the BBB may be involved in disease progression, eliciting of peripheral immune response, and, most importantly, altered drug efficacy. In this review, we will give a brief overview of the $\mathrm{BBB}$, its components, and their functions. We will critically evaluate the current literature in $\mathrm{AD}$ and $\mathrm{PD} \mathrm{BBB}$ pathology resulting from insult, neuroinflammation, and neurodegeneration. Specifically, we will discuss alterations in tight junction, transport and endothelial cell surface proteins, and vascular density changes, all of which result in altered permeability. Finally, we will discuss the implications of BBB dysfunction in current and future therapeutics. Developing a better appreciation of $\mathrm{BBB}$ dysfunction in $\mathrm{AD}$ and $\mathrm{PD}$ may not only provide novel strategies in treatment, but will prove an interesting milestone in understanding neurodegenerative disease etiology and progression [48].

Although intravenous lipid emulsion (ILE) was first used to treat life-threatening local anesthetic (LA) toxicity, its use has expanded to include both non-local anesthetic (non- LA) poisoning and less severe manifestations of toxicity. A collaborative workgroup appraised the literature and provides evidence-based recommendations for the use of ILE in poisoning.

Following a systematic review of the literature, data were summarized in four publications: LA and non-LA poisoning efficacy, adverse effects, and analytical interferences. Twenty-two toxins or toxin categories and three clinical situations were selected for voting. Voting statements were proposed using a predetermined format. A two-round modified Delphi method was used to reach consensus on the voting statements. Disagreement was quantified using RAND/ UCLA Appropriateness Method.

For the management of cardiac arrest, we recommend using ILE with bupivacaine toxicity, while our recommendations are neutral regarding its use for all other toxins. For the management of life-threatening toxicity, (1) as first line therapy, we suggest not to use ILE with toxicity from amitriptyline, non-lipid soluble beta receptor antagonists, bupropion, calcium channel blockers, cocaine, diphenhydramine, lamotrigine, malathion but are neutral for other toxins, (2) as part of treatment modalities, we suggest using ILE in bupivacaine toxicity if other therapies fail, but are neutral for other toxins, (3) if other therapies fail, we recommend ILE for bupivacaine toxicity and we suggest using ILE for toxicity due to other LAs, amitriptyline, and bupropion, but our recommendations are neutral for all other toxins. In the treatment of non-life-threatening toxicity, recommendations are variable according to the balance of expected risks and benefits for each toxin. For LA-toxicity we suggest the use of Intralipid $^{\star} 20 \%$ as it is the formulation the most often reported. There is no evidence to support a recommendation for the best formulation of ILE for non-LAs. The voting panel is neutral regarding ILE dosing and infusion duration due to insufficient data for non-LAs. All recommendations were based on very low quality of evidence.

Clinical recommendations regarding the use of ILE in poisoning were only possible in a small number of scenarios and were based mainly on very low quality of evidence, balance of expected risks and benefits, adverse effects, laboratory interferences as well as related costs and resources. The workgroup emphasizes that dose-finding and controlled studies reflecting human poisoning scenarios are required to advance knowledge of limitations, indications, adverse effects, effectiveness, and best regimen for ILE treatment [49].

Intralipid emulsion therapy is well-established for the treatment of local-anesthetic systemic toxicities. In recent years, its role has expanded as an important therapeutic agent in the reversal of other types of drug overdoses, including certain types of antipsychotics, antidepressants, antiarrhythmics, and calcium channel blockers. A literature review identified thirty-one case reports including fortynine separate drug overdose cases involving ten separate drug classes which were successfully reversed with Intralipid. The present clinical case study describes an elderly unresponsive woman refractory to conventional treatments after ingesting a potentially lethal amount of 5.6 grams of diltiazem in a suicide attempt. After treatment with Intralipid over a twenty-four hour period, the patient's hemodynamic and metabolic derangements were corrected and stabilized completely. Intralipid emulsion rescue therapy provides another potential strategy for the reversal of many drug toxicities, most likely by providing a lipid layer safety net for drug overdose by passive diffusion. Clinicians are urged to embrace an expanded role of Intralipid emulsion rescue therapy, not only for local anesthetic drug toxicities, but also for other lipophilic drug overdoses [50].

Caffeine is arguably the most widely used stimulant drug in the world. Here we describe a suicide attempt involving caffeine overdose whereby the patient's severe intoxication was successfully treated with the prompt infusion of Intralipid. A 19-year-old man was found in an agitated state at home by the volunteer emergency team about $1 \mathrm{~h}$ after the intentional ingestion of $40 \mathrm{~g}$ of caffeine (tablets). His consciousness decreased rapidly, followed quickly by seizures, and electrocardiographic monitoring showed ventricular fibrillation. Advanced life support maneuvers were started immediately, with the patient defibrillated 10 times and administered $5 \mathrm{mg}$ epinephrine 
in total and $300+150 \mathrm{mg}$ of amiodarone (as well as lidocaine and magnesium sulfate). The cardiac rhythm eventually evolved to asystole, necessitating the intravenous injection of epinephrine to achieve the return of spontaneous circulation. However, critical hemodynamic instability persisted, with the patient's cardiac rhythm alternating between refractory irregular narrow complex tachycardia and wide complex tachycardia associated with hypotension. In an attempt to restore stability we administered three successive doses of Intralipid $(120+250+100 \mathrm{mg})$, which successfully prevented a severe cardiovascular collapse due to a supra-lethal plasma caffeine level (>120 mg/L after lipid emulsion). The patient survived without any neurologic complications and was transferred to a psychiatric ward a few days later. The case emphasizes the efficacy of intravenous lipid emulsion in the resuscitation of patients from non-local anesthetic systemic toxicity. Intralipid appears to act initially as a vehicle that carries the stimulant drug away from heart and brain to less wellperfused organs (scavenging mechanism) and then, with a sufficient drop in the caffeine concentration, possibly as a tonic to the depressed heart [51].

Thrombosis and immune dysfunction are two important complications that result from the administration of parenteral nutrition. Endothelial cells within the vasculature are crucial components necessary for maintenance of normal coagulation and immune function.

We compared the effects of three commercial lipid emulsions (LEs; Intralipid ${ }^{\star}$, ClinOleic ${ }^{\circledR}$ [or Clinolipid ${ }^{\star}$ ], and Omegaven ${ }^{\star}$ ) differing in the levels of omega- 6 polyunsaturated fatty acids, omega-3 polyunsaturated fatty acids, omega- 9 monounsaturated fatty acids, and saturated fatty acids upon endothelial cell fatty acid composition using Gas chromatography, endothelial cell integrity by assessing measurement of apoptosis and necrosis using flow cytometry, endothelial cell inflammatory activation by assessing the induction of ICAM-1 by lipopolysaccharide [LPS]), and transcription factor activation (phosphorylation of NF- $\mathrm{KB}$ ) using western blot analysis.

Gas chromatographic analysis confirmed cellular uptake of the fatty acids within the LEs; furthermore, these fatty acid changes reflected the composition of the oils and egg phosphatides used in the manufacturing of these emulsions. However, the kinetics of fatty acid uptake and processing differed between LEs. Fish oil LE negatively impacted cell viability by doubling the percentage of apoptotic and necrotic cell populations quantified by flow cytometry using Annexin V/Fluorescein and propidium iodide. The soybean oil LE did not alter cell viability, while the olive oil-predominate emulsion improved cell viability. All LEs were capable of suppressing LPS-induced ICAM-1 expression; however, the fish oil LE was more potent than the other emulsions. Fish oil LE supplementation of cells also suppressed LPS-induced phosphorylation of NF- $\kappa \mathrm{B}$, while the soybean oil and olive predominant LE had no effect upon NF- $\kappa B$ phosphorylation. Lipid emulsions are readily incorporated and stored in the form of triacylglycerols. Soybean oil-based, olive oil-predominant and fish-oil based LEs differentially affected endothelial cell integrity. Importantly, these three LEs were capable of suppressing endothelial cell inflammatory response despite their fatty acid content [52]. Membrane currents conducted by the NMDA receptor channels were investigated in cultured cortical neurons and TsA cells transfected with NR1-1a/ NR2A subunits of the NMDA receptor. The whole-cell recording technique was used. Current transients evoked by bath application of NMDA for $5 \mathrm{~s}$ were characterized by a fast peak and a slow decay to $46.1+/-15.5 \%$ of the peak level at the end. When NMDA was applied in combination with various lipid emulsions (Intralipid, ClinOleic, Lipofundin or Abbolipid, the NMDA- induced currents were reduced, although this reduction did not affect the fast peak, it did affect the decay phase. The amount of reduction depended on the concentration of the lipids (in the case of Abbolipid diluted at 1:40, the current at the end of the 5-s drug application was approximately $2 / 3$ of control). When Abbolipid was applied $40 \mathrm{~s}$ before NMDA, peak and late current were reduced to approximately $2 / 3$. The effect of current reduction was the same at either of the two chosen membrane potentials $(-80$ and $+40 \mathrm{mV}$ ) which indicates that the effect was not mediated by contamination of the emulsions with $\mathrm{Mg}(2+)$. The current reduction produced by Abbolipid was about the same in native neuronal cells and in TsA cells expressing the NR1-1a/NR2A subunits. The current reducing effect of the lipid emulsions may add to the anesthetic, analgesic and neuroprotective effects seen with hypnotics administered by way of lipid carriers [53]. Little is known about the impact of circulating lipids on brain processes. Building on evidence that chronic fat consumption stimulates hypothalamic peptides in close association with elevated triglycerides (TG), this study examined whether an acute rise in TG levels induced by fat emulsion can affect these hypothalamic systems. In normal weight rats, ip injection of Intralipid $(20 \%, 5 \mathrm{ml})$ during the first $4 \mathrm{~h}$ after injection produced a robust increase in TG levels and nonesterified fatty acids, but had no impact on glucose, insulin, or leptin levels. This was accompanied by a marked increase in the expression of particular orexigenic peptides, galanin, orexins, and the opioid, enkephalin, which are known to be positively related to fat ingestion. This effect, similarly induced by $4 \mathrm{~h}$ of high fat diet consumption, was detected in the paraventricular nucleus (PVN) for galanin, in the perifornical hypothalamus (PFH) for orexins, and in the PVN, PFH, as well as the arcuate nucleus (ARC) for enkephalin. It was not seen, however, for neuropeptide $\mathrm{Y}$ and agouti-related protein localized in the ARC, which are unaffected or reduced by dietary fat. This site specificity was confirmed by c-Fos immunostaining, a marker of neuronal activity, which was increased by Intralipid in the PVN and $\mathrm{PFH}$, but not in the ARC, and was detected in $20 \%$ of orexin-expressing neurons in the PFH. These findings suggest that circulating lipids, through different mechanisms, may stimulate hypothalamic neurons, which synthesize specific feeding stimulatory peptides that possibly contribute to hyperphagia during consumption of a fat-rich diet [54].

\section{Conclusion}

Intralipid treatment is first suggested here for the treatment of Alzheimer disease. It should be given intravenously on a monthly basis according to each patient's response. Clinical studies should be done in order to evaluate this new treatment modality

\section{References}

1. Alzheimer's association (2017) Treatments for Alzheimer's.

2. Berrios G E (1991) Alzheimer's Disease: A Conceptual History. International Journal of Geriatric Psychiatry 5: 355-365.

3. Engstrom, Eric J (2007) Researching Dementia in Imperial Germany: Alois Alzheimer and the Economies of Psychiatric Practice. Culture, Medicine and Psychiatry 31: 405-413.

4. https://en.wikipedia.org/wiki/Alois_Alzheimer

5. Zipser BD, Johanson J, Gonzalez L, Berzin TM, Tavares R, et al. (2007) Microvascular injury and blood-brain barrier leakage in Alzheimer's disease. Neurobiology of Aging 28: 977-86. [Crossref]

6. Nagele, Robert G (2006) Alzheimer's disease: new mechanisms for an old problem UMDNJ Research. University of Medicine and Dentistry of New Jersey 7: 2.

7. https://webbeta.archive.org/web/20110917001430/http://www.umdnj.edu/research/ publications/fallo 6/4.htm 
8. Daviglus ML, Bell CC, Berrettini W (2010) National Institutes of Health state-of-thescience conference statement: preventing Alzheimer disease and cognitive decline. Annals of Internal Medicine 153: 176-181. [Crossref]

9. World Health Organization and Alzheimer's Disease International, Dementia (2012) A Public Health Priority.

10. David MS, Ann MS, Shi DY, Berislav Z (2000) Method to increase cerebral blood flow in amyloid angiopathy.

11. Russell RL, Westfall BA (1962) Alleviation of barbiturate depression. Anesth Analg 41: 582-585. [Crossref]

12. Krieglstein J, Meffert A, Niemeyer D (1974) Influence of emulsified fat on chlorpromazine availability in rabbit blood. Experientia 30:924-926. [Crossref]

13. Straathof DJ, Driessen O, Meijer JW, Van Rees H, Vermeij P, et al. (1984) Influence of Intralipid infusion on the elimination of phenytoin. Arch Int Pharmacodyn Ther 267: 180-186. [Crossref]

14. Weinberg GL, VadeBoncouer T, Ramaraju GA, Garcia-Amaro MF, Cwik MJ (1998) Pretreatment or resuscitation with a lipid infusion shifts the dose-response to bupivacaine-induced asystole in rats. Anesthesiology 88: 1071-1075. [Crossref]

15. Weinberg G (2002) Current concepts in resuscitation of patients with local anesthetic cardiac toxicity. Reg Anesth Pain Med 27: 568-575. [Crossref]

16. Cave G, Harvey M (2009) Intravenous lipid emulsion as antidote beyond local anesthetic toxicity: a systematic review. Acad Emerg Med 16: 815-824. [Crossref]

17. Rosenblatt MA, Abel M, Fischer GW, Itzkovich CJ, Eisenkraft JB (2006) Successful use of a $20 \%$ lipid emulsion to resuscitate a patient after a presumed bupivacainerelated cardiac arrest. Anesthesiology 105: 217-218. [Crossref]

18. Weinberg G, Di Gregorio G, Hiller D, Hewett A, Sirianni A (2009) Reversal of haloperidol-induced cardiac arrest by using lipid emulsion. Ann Intern Med 150: $737-$ 738. [Crossref]

19. Minton NA, Goode AG, Henry JA (1987) The effect of a lipid suspension on amitriptyline disposition. Arch Toxicol 60: 467-469. [Crossref]

20. Cave G, Harvey M, Graudins A (2011) Review article: Intravenous lipid emulsion as antidote: A summary of published human experience. Emerg Med Australas 23: 123141. [Crossref]

21. Tomiyama $T$ (2010) Involvement of beta-amyloid in the etiology of Alzheimer's disease. Brain Nerve 62: 691-699. [Crossref]

22. Bloom GS (2014) Amyloid- $\hat{I}^{2}$ and tau: the trigger and bullet in Alzheimer disease pathogenesis. JAMA Neurol 71: 505-508. [Crossref]

23. Zetterberg H, Mattsson N (2014) Understanding the cause of sporadic Alzheimer's disease. Expert Rev Neurother 14: 621-630. [Crossref]

24. Morris GP, Clark IA, Vissel B (2014) Inconsistencies and controversies surrounding the amyloid hypothesis of Alzheimer's disease. Acta Neuropathol Commun 2:135. [Crossref]

25. James AP, Pal S, Gennat HC, Vine DF, Mamo JC (2003) The incorporation and metabolism of amyloid-beta into chylomicron-like lipid emulsions. J Alzheimers Dis 5: 179-188. [Crossref]

26. Champeil PG, Denis I, Goustard LB, Alessandri JM, Guesnet P, et al. (2004) Astrocytes in culture require docosahexaenoic acid to restore the $n-3 / n-6$ polyunsaturated fatty acid balance in their membrane phospholipids. J Neurosci Res 75: 96-106. [Crossref]

27. Langelier B, Linard A, Bordat C, Lavialle M, Heberden C (2010) Long chain-polyunsaturated fatty acids modulate membrane phospholipid composition and protein localization in lipid rafts of neural stem cell cultures. J Cell Biochem 110: 1356-64. [Crossref]

28. Colin J, Gregory PL, Lanhers MC, Claudepierre T, Corbier C, et al. (2016) Membrane raft domains and remodeling in aging brain. Biochimie 130: 178-187. [Crossref]

29. Taftachi F, Sanaei ZH, Sepehrian B, Zamani N (2012) Lipid emulsion improves Glasgow coma scale and decreases blood glucose level in the setting of acute non-local anesthetic drug poisoning--a randomized controlled trial. Eur Rev Med Pharmacol Sci 1:38-42. [Crossref]

30. Weigt HU, Georgieff M, Beyer C, Föhr KJ (2002) Activation of neuronal N-methyl-Daspartate receptor channels by lipid emulsions. Anesth Analg 94: 331-337. [Crossref]

31. Haywood SC, Bree AJ, Puente EC, Daphna-Iken D, Fisher SJ (2009) Central but not systemic lipid infusion augments the counterregulatory response to hypoglycemia. $\mathrm{Am}$ J Physiol Endocrinol Metab 297: E50-E60. [Crossref]

32. Hiller DB, Di Gregorio G, Kelly K, Ripper R, Edelman L, et al. (2010) Safety of high volume lipid emulsion infusion: a first approximation of LD50 in rats. Reg Anesth Pain Med 35: 140-144. [Crossref]
33. Heinonen JA, Litonius E, Backman JT, Neuvonen PJ, Rosenberg PH (2013) Intravenous lipid emulsion entraps amitriptyline into plasma and can lower its brain concentration-an experimental intoxication study in pigs. Basic Clin Pharmacol Toxicol 113: 193 200. [Crossref]

34. Basarslan SK, Alp H, Senol S, Evliyaoglu O, Ozkan U (2014) Is intralipid fat emulsion a promising therapeutic strategy on neurotoxicity induced by malathion in rats? Eur Rev Med Pharmacol Sci. 18: 471-476. [Crossref]

35. Ozkan U, Osun A, Basarslan K, Senol S, Kaplan I, et al. (2014) Effects of intralipid and caffeic acid phenethyl ester on neurotoxicity, oxidative stress, and acetylcholinesterase activity in acute chlorpyriphos intoxication. Int J Clin Exp Med 7:837-46. [Crossref]

36. Heinonen JA, Litonius E, Salmi T, Haasio J, Tarkkila P, et al. (2015) Intravenous lipid emulsion given to volunteers does not affect symptoms of lidocaine brain toxicity. Basic Clin Pharmacol Toxicol 116: 378-83. [Crossref]

37. Lange DB, Schwartz D, DaRoza G, Gair R (2012) Use of intravenous lipid emulsion to reverse central nervous system toxicity of an iatrogenic local anesthetic overdose in a patient on peritoneal dialysis. Ann Pharmacother 46: e37. [Crossref]

38. http://ecatalog.baxter.com/ecatalog/loadResource.blob?bid=20000307

39. Gilbert DP, Tae WK (2011) Linking lipids to Alzheimer's disease: cholesterol and beyond. Nature Reviews Neuroscience 12: 284-296. [Crossref]

40. Terracina L, Brunetti M, Avellini L, De Medio GE, Trovarelli G, Gaiti A (1992) Linoleic acid metabolism in brain cortex of aged rats. The Italian Journal of Biochemistry 41:225-235. [Crossref]

41. Thomas MH, Pelleieux S, Vitale N, Olivier JL (2016) Dietary arachidonic acid as a risk factor for age-associated neurodegenerative diseases: Potential mechanisms. Biochimie 130: 168-177. [Crossref]

42. Karczewska KM, Kowalska I, Niko§̊,ajuk A, Adamska A, ZieliÅska M, et al. (2012) Circulating brain-derived neurotrophic factor concentration is downregulated by intralipid/heparin infusion or high-fat meal in young healthy male subjects. Diabetes Care 35: 358-362. [Crossref]

43. Rodriguez-Navas C, Morselli E, Clegg DJ (2016) Sexually dimorphic brain fatty acid composition in low and high fat diet-fed mice. Mol Metab 5: 680-689. [Crossref]

44. Sharma HS, Castellani RJ, Smith MA, Sharma A (2012) The blood-brain barrie in Alzheimer's disease: novel therapeutic targets and nanodrug delivery. Int Rev Neurobiol 102: 47-90. [Crossref]

45. Desai BS, Monahan AJ, Carvey PM, Hendey B (2007) Blood-brain barrier pathology in Alzheimer's and Parkinson's disease: implications for drug therapy. Cell Transplant 16: 285-299. [Crossref]

46. Zlokovic BV (2002) Vascular disorder in Alzheimer's disease: role in pathogenesis of dementia and therapeutic targets. Adv Drug Deliv Rev 54: 1553-1559. [Crossref]

47. Bartels AL (2011) Blood-brain barrier P-glycoprotein function in neurodegenerative disease. Curr Pharm Des 17: 2771-2777. [Crossref]

48. Desai BS, Monahan AJ, Carvey PM, Hendey B (2007) Blood-brain barrier pathology in Alzheimer's and Parkinson's disease: implications for drug therapy. Cell Transplant 16: 285-299. [Crossref]

49. Gosselin S, Hoegberg LC, Hoffman RS (2016) Evidence-based recommendations on the use of intravenous lipid emulsion therapy in poisoning. Clin Toxicol (Phila) 54: 899-923. [Crossref]

50. Muller SH, Diaz JH, Kaye AD (2016) Intralipid Emulsion Rescue Therapy: Emerging Therapeutic Indications in Medical Practice. J La State Med Soc 168: 101-103. [Crossref]

51. Muraro L, Longo L, Geraldini F, Bortot A, Paoli A, et al. (2016) Intralipid in acute caffeine intoxication: a case report. J Anesth 30: 895-899. [Crossref]

52. Harvey KA, Xu Z, Pavlina TM, Zaloga GP, Siddiqui RA (2015) Modulation of endothelial cell integrity and inflammatory activation by commercial lipid emulsions. Lipids Health Dis 18: 14-19. [Crossref]

53. Weigt H, Georgieff M, Beyer C, Georgieff EM, Kuhse J, et al. (2004) Lipid emulsions reduce NMDA-evoked currents. Neuropharmacology 47: 373-380. [Crossref]

54. Chang GQ, Karatayev O, Davydova Z, Leibowitz SF (2004) Circulating triglycerides impact on orexigenic peptides and neuronal activity in hypothalamus. Endocrinology 145: 3904-3912. [ ].

Copyright: (C2017 Eldor J. This is an open-access article distributed under the terms of the Creative Commons Attribution License, which permits unrestricted use, distribution, and reproduction in any medium, provided the original author and source are credited. 